\title{
GW23-e0867 VASCULAR CALCIFICATION INCREASES RISK OF HARD CORONARY HEART DISEASES IN THE GENERAL POPULATION OF SOUTH CHINA: A CASE CONTROL STUDY
}

doi:10.1136/heartjnl-2012-302920d.17

Huang Hui, Wang Jingfeng. Department of Cardiology, Sun Yat-Sen Memorial Hospital of Sun Yat-Sen University

Objectives Vascular calcification (VC) is regarded as an important cardiovascular risk maker and the relevant mechanisms are still under debate. The aim of this study is to evaluate the association between VC and risk of severe coronary events in the general population of South China, and identify the risk factors and predictors of VC.

Methods This study recruited 1604 people without coronary heart disease and diabetes mellitus in South China undergoing ankle-brachial index $(\mathrm{ABI})$ test. 38 members were defined as calcification group $(\mathrm{ABI} \geq 1.3)$ and 43 members were randomly selected from all the members with normal $\mathrm{ABI}(0.9<\mathrm{ABI}<1.3)$ as control group. Biochemical parameters and clinical characteristics were compared between these two groups.

Results The Framinghan 10-year hard coronary heart disease (HCHD) risk of the calcification group was significant higher than the control group $(9.20 \pm 4.21 \%$ vs $3.21 \pm 2.07 \%, p<0.001)$. Compared with the control group, the morbidities of hypertension and ischaemic stroke were higher in the calcification group $(62.5 \%$ vs $38.1 \%, p<0.05$ and $21.9 \%$ vs $2.4 \%, p<0.01$ ). In an adjusted model of binary logistic regression, the OR was 1.185 for BMI and 1.194 for hsCRP (95\% CI 1.002 to 1.319 and 1.045 to 1.420 ). Both of these indexes were the independent risk factors for VC $(p<0.05)$. The areas under the receiver operating characteristic (ROC) curve were 0.629 for BMI and 0.723 for hsCRP. Both BMI and hsCRP were effective predictors for VC $(p<0.05)$.

Conclusions VC associated with inflammation and obesity increases the risk of HCHD in general population of South China. 